\title{
श्री कृष्ण से संबंधित ख्याल रचनाओं एवं बंदिशों का मानवीय जीवन पर प्रभाव \\ ज्योति शर्मा
}

शोध छात्रा, संगीत विभाग, पंजाब विश्वविद्यालय, चंडीगढ

\begin{abstract}
Lord Krishna is the most popular God in whole India. Many famous saints wrote bhajans in the praise of Load Krishna. Kord Krishna has many names and devotees chant different names of Krishna. In Indian classical music many compositions are found in which life, work and other aspects of Krishna are there. These song/composition has a great impact on the life of Indian people. Bhakti filled compositions are most popular in the society.
\end{abstract}

Key words : Krishna, Classical Compositions, Classical Raga.

भारतीय संस्कृति का सबसे प्राचीन एवं प्रमुख धार्मिक हिन्दू धर्म है। वैदिक आर्य संस्कृतिक के अनुरूप ही हिन्दू धर्म का विकास हुआ। आगे चलकरर इस धर्म में शैव-शक्त परम्पराएँ एवं मध्यकारल में वैष्णव सम्प्रदाय स्थापित हुए है। इन्होंने भी अपनी धार्मिक पूजा-अर्चना विधियों में संगीत का विपुल प्रयोग किया।

"भक्ति और साधना का भाव मानव मात्रा पाया जाता है। भारत तो वैसे भी धर्म और सम्प्रदाय अवतारों में राम और कृष्ण का महत्त्व अक्षुण्ण है। कृष्णावतार की कल्पना पुरानी और व्यापक है। ${ }^{\prime \prime 1}$ इसका कारण कृष्ण के व्यक्तित्व और बहुआयामी होना है। उनका व्यक्तित्व महान, व्यापक आकर्षक और मधुर है कि भारतीय जनमानस को किसी भी मनुष्य, अवतार या देवी देवता का व्यक्तित्व उतना प्रभावित नहीं करता जितना कृष्ण का। "उनकी विविध असंख्या लीलाओं में एक मानवीय रस, इसी कारण उनका चरित सार्वभौम आकर्षण का केन्द्र बन गया है $\left.\right|^{\prime \prime 2}$

कृष्ण भारतीय संस्कृति के सबसे बहुरंगी व्यक्तित्व है। श्री कृष्ण भारत के गौरव के आधर स्तभ है। कृष्ण का विस्तृत क्षेत्र होने के कारण समय-समय पर कृष्ण के स्वरूप में भी अंतर आता गया। महाभारत के कृष्ण आज के कृष्ण से सर्वथा भिन्न प्रतीत होते है अतः श्री कृष्ण का स्वरूप भी उन्हीं के अनुरूप परिवर्तित होता है।

भगवान श्री कृष्ण का कथन है वेदानां सामवेदोऽस्मि अर्थात वेदों में मैं सामवेद हूँ। भगवान के हाथ में वेणु है। कृष्ण का कोई भी रूप हो वे सदा सर्वदा आराध्य है। पूजा की कोई भी क्रिया संगीत विहीन नहीं होती। भगवान श्री कृष्ण के सभी रूपों का विवरण जैसे बाल कृष्ण गोपी बिहारी श्री कृष्ण संबंधी जो गीत है उनका भारतीय साहित्य और संगीत में बहुत प्रभाव पड़ा।

1 हिन्दी साहित्य, आचार्य हजारी प्रसाद द्विवेदी, पृ. 92 ।

2 जैन हिम्मत सिंह, हिन्दी कृष्ण चरित्र काव्य, पृ. 09। 
भारतीय साहित्य, संगीत कला और धर्म सभी में कृष्ण का आकर्षक व्यक्तित्व विविध रूपों में अपनाया गया है। वह वस्तुतः महानता का पूर्ण निदर्शन है: ज्ञान में महान, बुद्धि और मस्तिष्क की कृतियों को महानतम लगने वाली चारित्रिक विशेषताओं का पूर्णधार है। वे अपने युग की महानतम विभूति और आगामी युगों की अज्ञय उत्स है।

\section{"वेदानां सामेवेदोडिस्म देवानामस्मि वास्वः \\ इन्द्रियाणां मनस्मि भूतानास्मि चेतना।।"1}

मैं वेदों में सामवेद हूँ। इन्द्रियों में मन हूँ, भूत प्राणियों में चेतना अर्थात् ज्ञान हूँ। भगवान श्री कृष्ण ने अर्जुन को कहा वेदों में मैं सामवेद हूँ। कृष्ण शब्द और सिद्धांत और शास्त्र में में नहीं हूँ। गीत, संगीत और नृत्य में में हूँ और जीवन का परम रहस्य, वह तो तल्लीनता में पूरा होता है। सामवेद तल्लीनता का शास्त्र है इसलिए भगवान ने ऋग्वेद को नहीं सामवेद को कहा है। भगवान की विभूतियाँ अनंत है। जो कुछ भी इस जगत में होता है वह भगवान की मर्जी के बिना नहीं हो सकता। अच्छा और बुरा भी मैं होने देता हूँ तभी होता है। भगवान ने अंत में विभूति योग दसवां अध्याय में कहा है कि यह सारा जगत मेरी विभूति के अंशमात्र से स्थित है।

\section{संगीत से संबंध}

भगवान श्री कृष्ण का संगीत से संबंध प्रत्येक काल में रहा है। कृष्ण जो कि सोलह कला सम्पूर्ण माने जाते है समस्त भारत में उपास्य देव प्रतिष्ठित हुए।

"मध्यकाल को भक्ति काल माना गया है। सगुण तथा निर्गुण भक्ति की दो धरायें प्रवाहित हुई। सगुण भक्ति धरा में राम तथा कृष्ण का गुणगान हुआ। तुलसीदास जी ने 'रामचरित मानस' नामक अमर गेय काव्य की रचना की तो सूर, मीरा, आदि अष्टछाप के कवियों ने कृष्ण की स्तुति में विभिन्न काव्य रचना कर गान किया। इसका प्रभाव शास्त्रीय संगीत की रचनाओं पर भी प्रभाव पड़ा। सूर जैसा भाव, मीरा जैसा प्रेम और तुलसी जैसी श्रद्धा रखकर भक्ति संगीत प्रस्तुत किया जाए, तो मनुष्य का जीवन सफल हो जाए। ${ }^{\prime 2}$ मुरलीधर श्रीकृष्ण से संबंधित आधुनिक काल तक विभिन्न कवि या गीतकार लिखते चले आए हैं।

\section{शैलियाँ}

श्रीकृष्ण से संबंधित सांगीतिक रचनाएँ एवं शैलियाँ जैसे सामगान, गाया, धुवा, जाति, प्रबंध, ध्रुपद, धमार एवं ख्याल की पद, गीत प्रचार में है। कृष्ण संबंधी जो गीत प्राप्त होते हैं, उनका भारतीय गीत साहित्य और संगीत में बड़ा प्रभाव पड़ा अर्थात कृष्ण की मुरली का जो विवरण इन गीतकाव्यों से आरम्भ हुआ, वह आज तक भारतीय संगीत एवं साहित्य में अवरूप रूप से चले आ रहे हैं। यशोदा की वात्सल्य भक्ति, वेणु गीत में कृष्ण की मुरली की मधुर तान, गोपिका गीत में भी श्री कृष्ण और गोपियों का श्रृंगारिक विवरण भ्रमरगीत में गोपियों का कृष्ण के प्रति अट्ट प्यार इन सभी संदर्भों में जो कृष्ण के रूप का गान और उनकी मुरली की तान की गीत काव्य में सांगीतिक व्याख्या की गई है। मुरलीधर श्री कृष्ण से सम्बंधित

1 श्रीमद्भगवतगीता, श्लोकार्थसहित, गीता प्रेस, 10वां अध्याय पृ. 133 ।

2 भक्ति संगीत अंक, लक्ष्मी नारायण गर्ग, हाथरस, पृ. 6 । 
आधुनिक काल तक विभिन्न गीतकार, वाग्ग्येकार लिखते चले आए है। ख्याल गायक भी प्रायः मुरलीधर श्रीकृष्ण से संबंधित ख्याल की बंदिश जब गाते है तो उसमें मुरली वाद्य का विवरण अवश्य प्राप्त होता है।

\section{विषय}

कृष्ण से संबंधित ख्याल रचनाओं का विषय के अन्तर्गत लीला, होरी, प्रेम, रास, श्रृंगार, दर्शन, अध्यात्म और कर्मयोग में भगवान श्री कृष्ण की नाना प्रकार की लीलाएँ, होरी गीत जो कि संगीत के शास्त्रीय और व्यवहारिक क्रम में बंधे हुए धमार, दीपचंदी इत्यादि तालों रचित मिलते हैं जिसका विषय प्रेम, होरी संबंधी गीतों में होता है। वेणु गीत, गोपिका गीत, युगल गीत, भ्रमर गीत, रास इत्यादि कषष्ण संबंधी बंदिशों का वर्णन मिलता है जो कि भावात्मक और अनुकूल वातावरण का दृश्य दृष्टिगोचर होता है। ख्याल में प्रयुक्त राग एवं ताल इत्यादि की परम्परागत बंदिशों में नियमों का पालन किया जाता है।

\section{रस}

कृष्ण परक ख्याल बंदिशों में श्रृंगार रस, भक्ति रस, वात्सलय रस, वीर रस की रचनाओं का उल्लेख मिलता है। भाव में रस है और रस में भाव है। मन की अन्तरदृष्टि को दर्शाने के भाव महत्त्वपूर्ण भूमिका निभाते हैं। भावनाओं से रसों की उत्पत्ति का स्त्रोत कृष्ण परक ख्याल बंदिशों में दृष्टिगोचर होता है।

\section{मानवीय जीवन पर प्रभाव}

ख्याल के साहित्य में भक्ति अध्यात्मिक, श्रृंगार, लौकिक इत्यादि बंदिशें रची गई जिन्हें विभिन्न वाग्ययेकारों ने अपनी साधना से परिष्कृत कर समाज में प्रस्तुत किया। विद्यालयों, महाविद्यालयों में संगीत जब विषय के रूप में आया तो विभिन्न कलाकारों व वाग्ययेकारों ने एवं शिक्षकों ने विभिन्न प्रकार की कृष्ण परक बंदिशों का शिक्षण एवं प्रदर्शन किया जैसे औंकार नाथ ठाकुर द्वारा लिखित पुस्तक संगीताजली, रामाश्रय झा - अभिनव गीताँजली, विनायक राव पटवर्धन - राग विज्ञान, बलवंत राय भट्ट - भाव रंग लहरी, क्रमिक पुस्तक मालिका - पण्डित विष्णु नारायण भातखण्डे आदि द्वारा लिखित इन पुस्तकों में बन्दिशे कृष्ण परक ख्याल रचनाओं के रूप में मिलती है। इन पुस्तकों में कृष्ण परक बन्दिशों का संग्रह प्राप्त होता है। मौखिक परम्परा द्वारा इन बन्दिशों का प्रयोग किया जाता है।

\section{राग - हमीर (तीन ताल)}

स्थाई - स्मरण करूँ श्याम, सुखकंद को दरशन,

करूं सदा ब्रजचंद, जपुनाम नंद को।।

अंतरा - प्रिय लाल गोपवृंद को महाकाल

महाकाल फविंद को भावरंग भज भुवनानंद को।। ${ }^{1}$

1 बलवंत राय भट्ट, भावरंग लहरी, भा. 1, पृ. 58 । 


\section{राग - बिलावल (एक ताल)}

स्थाई- ते हरनाम नाहीं, सुमिरन करे नाहक

गये दीन खोयो।

अंतरा- अमृत बेल थड़ी बृख बिरखा

कर चतुराई गोयो। 1

\section{राग - खमाज (तीनताल)}

स्थाई- ते हरि नाम सुमिरन कीनो एक दिन रैन

अंतरा- सुमिरन भजन करो दीन। केशव को अभय दान ताहे दीन। ${ }^{2}$

शास्त्रीय संगीत ताल बद्ध होने के कारण सामान्य गुण इससे वंचित रह जाते है। उसका प्रमुख कारण यह है कि शास्त्रीय संगीत का ज्ञान सामान्य वर्ग को न होने के कारण यह उनकी समझ में कुछ कम आता है। यदि इन बंदिशों का प्रयोग सुगम संगीत अर्थात भजन इत्यादि के रूप में किया जाये तो यह हर वर्ग के लोगों के द्वारा समझा जा सकता है।

आज समाज में जब सब तरफ अशांत वातावरण व्याप हो रहा है भौतिकता की दौड़ में सभी प्रभावित हो रहे और नैतिक मूल्यों का पतन होता दिखाई दे रहा है। आज विद्यालयों, महाविद्यालयों में जो ख्याल रचनाएँ एवं बंदिशें जो सिखाई जाती है वह अधिकतर घरेलू झगड़े जैसे सास बहू, ननद, सौतन इत्यादि विषयों पर आधरित है जबकि होना ऐसा चाहिए कि भक्ति परक बंदिशों को अनुकरण करना चाहिए। जिससे इन ख्याल रचनाओं का प्रदर्शन एवं प्रचार-प्रसार भी होगा जो कि भावी संगीत विद्यार्थियों एवं समाज के लिए कल्याणप्रद होगा।

\section{संदर्भ ग्रंथ सूची}

गुप्ता उषा, (2006) हिन्दी कृष्ण भक्तिकालीन साहित्य में संगीत, लखनऊ विश्वविद्यालय, लखनऊ।

नगेन्द्र, (1974) रस सिद्धांत, नेशनल पब्लिशिंग हाऊस दिल्ली।

शर्मा, डॉ. सुनीता (1996) भारतीय संगीत का इतिहास, संजय प्रकाशन दिल्ली।

गैशेला वाचस्पति (1973) भारतीय संस्कृति और कला, हिन्दी ग्रंथ अकादमी, लखनऊ।

शर्मा पंकज माला (1996) सामगानः उद्भव व्यवहार एवं सिद्धांत, कान्यायन वैदिक साहित्य प्रकाशन होशियारपुर।

शास्त्री देवट्टि (2009) कलानाथ संस्कृत साहित्य का इतिहास, धमाणी मार्किट की गलि, चौड़ा रास्ता, जयपुर संस्करण।

श्री मदभगवद्गीता प्रेस गोरखपुर।

श्री मद्भागवतमहापुराण महर्षि वेदव्यास, गीताप्रेस, गोरखपुर।

1 विनायक राव पटवर्धन, राग विज्ञान भा. 3, पृ. 156 ।

2 क्रमिक पुस्तक मालिका. भाग. 1, पृ. 10 । 